\title{
Évaluation des processus : comprendre comment le contexte des entreprises influence le déroulement des premières étapes d'interventions ergonomiques
}

Process evaluation: understanding how the organization's context influences the first steps of ergonomic interventions

Valérie Albert, Nicole Vézina, Henriette Bilodeau et Fabien Coutarel

\section{(2) OpenEdition}

Édition électronique

URL : http://journals.openedition.org/activites/3411

DOI : 10.4000/activites.3411

ISSN : $1765-2723$

Éditeur

ARPACT - Association Recherches et Pratiques sur les ACTivités

Référence électronique

Valérie Albert, Nicole Vézina, Henriette Bilodeau et Fabien Coutarel, «Évaluation des processus : comprendre comment le contexte des entreprises influence le déroulement des premières étapes d'interventions ergonomiques », Activités [En ligne], 15-2 | 2018, mis en ligne le 15 octobre 2018, consulté le 19 avril 2019. URL : http://journals.openedition.org/activites/3411 ; DOI : 10.4000/ activites.3411

Ce document a été généré automatiquement le 19 avril 2019

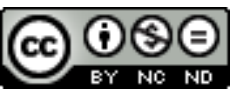

Activités est mis à disposition selon les termes de la licence Creative Commons Attribution - Pas d'Utilisation Commerciale - Pas de Modification 4.0 International. 


\section{Évaluation des processus :} comprendre comment le contexte des entreprises influence le déroulement des premières étapes d'interventions ergonomiques

Process evaluation: understanding how the organization's context influences the first steps of ergonomic interventions

Valérie Albert, Nicole Vézina, Henriette Bilodeau et Fabien Coutarel

\section{NOTE DE L'ÉDITEUR}

Article soumis le 3 septembre 2017, accepté le 20 janvier 2018

\section{Remerciements :}

Dans le cadre de son doctorat, Valérie Albert bénéficie du soutien financier des Instituts de recherche en santé du Canada (IRSC) et de l'Institut de recherche Robert-Sauvé en santé et sécurité du travail (IRSST).

\section{Introduction}

1 Les troubles musculosquelettiques (TMS) sont des problèmes de santé d'origine multifactorielle, fréquents et coûteux. Les interventions de prévention sont donc essentielles et l'ergonomie est une discipline de première ligne en ce qui concerne la prévention des TMS en milieu de travail. Cependant, il existe différentes approches d'intervention en ergonomie et on retrouve donc, à l'intérieur de la discipline, toute une gamme d'interventions, allant des plus simples aux plus complexes. L'évaluation des 
interventions ergonomiques visant la prévention des TMS devient donc utile pour identifier quelles sont les interventions les plus efficaces, mais également pour comprendre comment ces interventions fonctionnent et contribuent aux effets observés. Cette connaissance permet de mieux identifier comment améliorer ces interventions. Or, différentes approches d'évaluation existent, mais elles ne conviennent pas toutes à l'évaluation des interventions complexes.

2 Dans les journaux scientifiques internationaux du domaine de l'ergonomie, la grande majorité des évaluations ont été réalisées en ayant recours à la méthode expérimentale. Selon cette méthode, la population à l'étude est divisée de façon aléatoire en deux groupes: les individus $d u$ groupe expérimental reçoivent l'intervention sous investigation et les individus du groupe contrôle reçoivent soit une intervention placebo, soit l'intervention habituelle, et ce, dans le but de vérifier si l'intervention sous investigation produit des résultats significativement plus importants que le placebo ou l'intervention habituelle (Worrall, 2007). Or, pour maximiser la validité interne de l'évaluation, ou autrement dit pour s'assurer que les effets mesurés soient bien liés à l'intervention sous investigation, la méthode expérimentale exige d'une part d'annihiler tout effet lié au contexte : celui-ci est vu comme nuisible, un «bruit » qu'il faut contrôler afin d'isoler l'effet, lorsque présent, de l'intervention (Berthelette, Bilodeau, \& Leduc, 2008). En ayant recours à la méthode expérimentale, l'évaluation ne permet donc pas d'identifier de façon précise les indicateurs de contexte auxquels l'ergonome est confronté dans le cadre de son intervention (Diabaté, Stock, Vézina, Laberge, Albert, Sinden et al., 2016). D'autre part, toujours pour maximiser la validité interne de l'évaluation, la méthode expérimentale exige que l'intervention sous investigation soit la même pour tous les individus. Les interventions ergonomiques évaluées se limitent donc le plus souvent à des changements simples axés sur la réduction de facteurs de risque biomécaniques, par exemple un même équipement offert à un grand nombre de travailleurs, avec des effets mitigés sur la réduction des TMS.

3 Pourtant, il existe à l'intérieur de la discipline de l'ergonomie des approches d'intervention multifactorielles, centrées sur l'analyse de l'activité réelle de travail et visant l'amélioration des situations de travail en favorisant les interactions entre les acteurs (travailleurs et décideurs) et l'évolution de leurs représentations du travail concernant les déterminants des TMS. Les déterminants sont spécifiques à une situation de travail donnée et peuvent concerner l'organisation de la production, l'organisation de la formation, le dispositif technique, l'environnement social ou les tâches et exigences du travail (St-Vincent, Vézina, Laberge, Gonella, Lévesque, Petitjean-Roget, et al., 2010). Ces approches d'intervention, qui s'inscrivent dans le courant de l'ergonomie de l'activité (Daniellou, \& Rabardel, 2005), apparaissent donc très pertinentes au regard des modèles étiologiques multifactoriels des TMS. Cependant, ces interventions ergonomiques complexes peuvent être très différentes les unes des autres et très dépendantes du contexte des entreprises dans lesquelles ont lieu les interventions. Elles apparaissent donc peu compatibles aux exigences de la méthode expérimentale et se retrouvent très peu évaluées dans la littérature scientifique, à défaut d'un modèle adéquat pour le faire (Coutarel, \& Petit, 2015). Cependant, les interventions pour lesquelles des données probantes sont limitées ou inexistantes deviennent sous-estimées, même en présence de résultats prometteurs ou de mécanismes d'action plus solides (Vliet Vlieland, 2002). Il s'agit là d'un problème de taille: en l'absence de preuve de l'utilité sociale des interventions du courant de l'ergonomie de l'activité, on peut se demander pourquoi 
continuer à financer socialement ces interventions? Le développement d'un modèle d'évaluation adapté aux particularités des interventions du courant de l'ergonomie de l'activité apparaît donc prioritaire. C'est ce que nous proposons de faire dans le cadre de cette recherche interdisciplinaire.

\section{Cadre théorique}

4 Pour présenter le cadre théorique, nous utiliserons deux approches issues respectivement des disciplines de l'évaluation et de l'ergonomie :

1. L'évaluation des processus « fondée sur la théorie »;

2. Un modèle théorique initial de l'intervention ergonomique.

\section{1. Évaluation des processus pour mieux comprendre les interventions complexes}

Bien qu'il n'existe pas de frontière nette entre les interventions simples et les interventions complexes, les interventions complexes sont habituellement décrites comme des interventions qui :

1. Comportent plusieurs composantes qui interagissent les unes avec les autres;

2. Requièrent un haut niveau de flexibilité ou d'adaptation de l'intervention au contexte ;

3. Sont fournies à divers groupes d'acteurs de différents niveaux hiérarchiques;

4. Génèrent des effets diversifiés.

(Brousselle, Champagne, Contandriopoulos, \& Hartz, 2011 ; Craig, Dieppe, Macintyre, Michie, Nazareth, \& Petticrew, 2013 ; Moore, Audrey, Barker, Bond, Bonell, Hardeman, et al., 2015).

Lorsque l'on désire évaluer des interventions complexes dont la forme et la nature sont étroitement liées au contexte dans lequel elles se déroulent, il est souhaitable d'opter pour des devis permettant la description en profondeur du processus des interventions étudiées et l'appréciation qualitative des résultats en regard du contexte d'intervention (Coutarel, Vézina, Berthelette, Aublet-Cuvelier, Descatha, Chaissaing., et al., 2009). Une approche d'évaluation qui permet d'atteindre ces objectifs est l'évaluation des processus. En effet, alors qu'avec la méthode expérimentale, le contexte était perçu comme une source d'influence qu'il fallait contrôler, dans l'évaluation des processus le contexte est plutôt considéré comme une source d'explication du déroulement de l'intervention ou des effets (Brousselle et al., 2011), d'où sa pertinence pour nos travaux.

Or, la discipline de l'évaluation distingue deux grands types d'évaluation des processus : l'évaluation de la fidélité et l'évaluation "fondée sur la théorie» (Chen, 2005). L'évaluation de la fidélité mesure le degré de congruence entre, d'une part, les caractéristiques d'une intervention et des clientèles qu'elle rejoint, et d'autre part, ce qui avait été prévu (Chen, 2005 ; Moore et al., 2015). Ce type d'évaluation a déjà été utilisé dans le champ de l'ergonomie (Baumann, Holness, Norman, Idriss-Wheeler, \& Boucher, 2012 ; Dale, Jaegers, Welch, Gardner, Buchholz, Weaver, et al., 2016. ; Driessen, Proper, Anema, Bongers, \& van der Beek, 2010 ; Pehkonen, Takala, Ketola,, Viikari-Juntura, LeinoArjas, Hopsu, et al., 2009 ; Visser, van der Molen, Sluiter, \& Frings-Dresen, 2014) pour se prononcer sur la fidélité d'implantation d'interventions simples. Ces études avaient retenu un modèle théorique développé pour des interventions en santé publique qui 
mesure des variables comme le contexte, le recrutement, la portée de l'intervention, la dose d'intervention émise et la dose reçue, ainsi que la fidélité par rapport à ce qui avait été prévu au départ (Linnan, \& Steckler, 2002). Or, ce type d'évaluation des processus est fondé sur le postulat que la fidélité à ce qui était prévu est hautement désirable, mais ce postulat n'est pas universellement accepté : dans plusieurs situations, on reconnait qu'un écart avec ce qui a été prévu est souhaitable pour maximiser les retombées de l'intervention dans un contexte donné (Durlak, \& DuPre, 2008). Les interventions ergonomiques issues du courant de l'ergonomie de l'activité font d'ailleurs partie des interventions où des adaptations sont d'emblée prévues et souhaitables, puisqu'elles ne correspondent pas à l'application stricte d'un plan initialement conçu qui serait progressivement mis en œuvre (Petit, Querelle, \& Daniellou, 2007).

Dans de tels cas, il faut plutôt opter pour une évaluation des processus « fondée sur la théorie ", c'est-à-dire une évaluation systématique de la façon dont les composantes majeures d'une intervention sont prodiguées, en utilisant le modèle théorique de l'intervention comme cadre pour l'évaluation (Chen, 2005). Ce type d'évaluation comporte avant tout un volet descriptif et explicatif important, puisque son objectif est de comprendre le déroulement de l'intervention et l'enchaînement des événements (Brousselle et al., 2011; Langley, 2009). Comme les interventions complexes sont par nature imprévisibles et doivent être adaptées aux différents contextes (Moore et al., 2015), il est pertinent que l'évaluation permette de répondre à la question suivante : comment le contexte influence-t-il l'intervention et les effets obtenus? Concernant les effets, on distingue les effets finaux de l'intervention, comme la réduction du nombre de TMS parmi les travailleurs, des effets intermédiaires, qui consistent en des résultats produits pendant le déroulement de l'intervention, ou juste après la fin de l'intervention (Cole, Wells, \& "the worksite upper extremity research group", 2002 ; Landry, 2008). Des effets intermédiaires de l'intervention ergonomique sont, par exemple, les changements de représentation des acteurs-clés et le plan d'action décrivant les changements à implanter pour réduire les TMS et améliorer les situations de travail.

8 Le modèle théorique d'évaluation des processus (Nielsen, \& Randall, 2013) retenu dans le cadre de cette étude s'intéresse à trois paramètres :

1. Le contexte de l'entreprise, qui s'exprime sous forme de leviers ou d'obstacles ;

2. L'intervention, qui comprend les actions réalisées et les acteurs décisionnels impliqués ;

3. Les effets intermédiaires, comme les changements de représentations (perceptions des acteurs décisionnels) par rapport à l'intervention et le plan d'action qui décrit les changements à implanter.

Ce modèle théorique développé pour l'évaluation des processus d'interventions organisationnelles a été choisi, car il est compatible à une évaluation des processus «fondée sur la théorie » et présente des similarités intrinsèques avec les préoccupations des ergonomes concernant les paramètres de l'intervention à instruire pour en tirer des connaissances utiles pour bonifier les interventions futures. Dans le cadre de cet article, nous avons choisi de privilégier la comparaison des contextes d'intervention et des stratégies développées par les intervenants. Il est à noter que le niveau de détail quant au renseignement des stratégies peut être beaucoup plus affiné (Albert, 2018). Nous avons fait le choix de la concision dans cet article, car plusieurs recherches ont déjà fourni des descriptions très détaillées des stratégies d'intervention des ergonomes mais, en contrepartie, les généralisations possibles sont moins visibles, faute de catégorisation des indicateurs de contexte. Plus spécifiquement, nous nous concentrons sur les liens entre le 
contexte, le déroulement de l'intervention et les choix effectués par les acteurs-clés entourant le plan d'action. La question d'évaluation traitée dans cet article est donc la suivante : comment le contexte initial de l'entreprise a-t-il influencé le déroulement de l'intervention ergonomique et le plan d'action décrivant les changements à implanter pour prévenir les TMS? Le contexte correspond aux: «caractéristiques sociales, organisationnelles, politiques et culturelles des entreprises dans lesquelles les interventions sont implantées " (Berthelette et al., 2008). Dans le cadre de l'évaluation, nous nous intéressons donc au contexte interne de l'entreprise, puisque notre hypothèse est que les éléments du contexte externe à l'entreprise qui ont une influence sur l'intervention se manifesteront à l'interne. De plus, nous qualifions le contexte d'«initial», car nous admettons d'emblée qu'il est sujet à évoluer au cours de l'intervention ergonomique.

\subsection{Modèle théorique initial des interventions ergonomiques évaluées}

Évaluer une intervention passe par la description du modèle théorique initial de l'intervention, suivie de la description du déroulement réel de l'intervention (Coutarel et al., 2009). Les modèles théoriques initiaux correspondent aux différentes manières de transformer les situations de travail. Ces modèles permettent d'organiser l'action de l'ergonome, mais rappelons qu'ils ne visent pas à être appliqués : ils constituent un cadre initial et opérant qui doit être ajusté aux singularités des contextes et à leur dynamique non maitrisable (Coutarel et al., 2009). Comme exemples de modèles théoriques initiaux en ergonomie, certains intervenants pourraient privilégier le rôle de l'analyse du travail et la mise en circulation du "point de vue du travail» pour influencer les choix des acteurs-clés concernant les transformations à implanter (Guérin, Laville, Daniellou, Duraffourg, \& Kerguelen, 2007 ; St-Vincent et al., 2011). D'autres modèles vont privilégier le fait de s'insérer dans les structures de décision et de conception, les résultats de l'analyse du travail étant un matériau qui sera introduit à des moments stratégiquement choisis en vue d'influencer les processus de décision (Daniellou, 2004 ; Petit et al., 2007). Certains modèles ajoutent également une perspective développementale, où la conception des postes de travail ou la conduite du projet ne sont plus uniquement des finalités de l'action ergonomique, mais deviennent aussi des moyens pour développer le pouvoir d'agir des acteurs, afin de les rendre capables de réactualiser, lorsque nécessaire, leur connaissance du système et de gérer de futurs projets (Coutarel, Caroly, Vézina, \& Daniellou, 2015 ; Falzon, 2013). Les types de pratique peuvent également influencer le choix des modèles d'intervention, comme le consultant adoptant un modèle d'intervention ergonomique basé sur un système de relations de services (Querelle, \& Thibault, 2007). L'objectif n'est pas ici d'effectuer une revue exhaustive de l'ensemble des modèles d'intervention en ergonomie: il en existe une multitude. Cependant, dans le cadre d'une évaluation et plus particulièrement lorsque l'on souhaite comparer plusieurs interventions ergonomiques, il est souhaitable au plan méthodologique d'évaluer des interventions pour lesquelles les intervenants partagent un même modèle théorique initial.

10 Les interventions qui seront évaluées dans le cadre de notre étude se basent toutes sur un même un modèle théorique initial d'intervention en milieu de travail (St-Vincent et al., 2011), qui s'apparente au modèle développé par Guérin et al. (2007). Le modèle 
d'intervention de St-Vincent et al. (2011) sera donc élaboré dans les prochains paragraphes. Tout d'abord, ces auteurs (St-Vincent et al., 2011) définissent l'intervention ergonomique de la façon suivante: "l'intervention ergonomique est réalisée dans un contexte donné, à un moment donné, en vue de transformer les situations de travail pour les améliorer selon des critères de santé et d'efficacité. L'intervention implique la mise en œuvre d'un système organisé d'actions menées par l'ergonome en interaction avec des acteurs de l'entreprise et concerne non seulement le champ des changements techniques et organisationnels, mais se déploie aussi dans le champ social, touchant les acteurs et leurs représentations, notamment de la santé et du travail réel » (pages 64-65). Cette définition met en lumière que l'intervention ergonomique ne s'amorce pas au moment où les changements sont implantés, mais bien avant, soit dès le premier contact avec l'entreprise (de Montmollin, 1997). L'évaluation des processus réalisée dans le cadre de cette étude concerne donc la phase de l'intervention ergonomique qui précède l'implantation des changements, c'est-à-dire la phase au cours de laquelle l'ergonome procède à des analyses et au terme de laquelle le plan d'action de prévention est défini. Dans le domaine de l'évaluation, on réfère à cette phase comme la phase de développement de l'intervention (Goldenhar, LaMontagne, Katz, Heaney, \& Landsbergis, 2001). Des revues de littérature en ergonomie ont d'ailleurs souligné que la phase de développement des interventions ergonomiques est généralement très peu décrite, voire complètement absente des études évaluatives (Denis, St-Vincent, Imbeau, Jette, \& Nastasia, 2008 ; Yazdani et al., 2015), d'où la pertinence de cibler cette phase pour nos travaux.

11 Plus précisément, le modèle de St-Vincent et al. (2011) décrit deux grandes étapes à l'intérieur de cette première phase de développement de l'intervention qui précède l'implantation des changements, soit :

1. L'analyse de la demande ;

2. L'analyse des situations de travail menant au prédiagnostic ${ }^{1}$.

Pour compléter chaque étape, diverses actions sont réalisées auprès de nombreux acteurs de différents niveaux hiérarchiques en vue d'atteindre des balises (ou autrement dit des sous-objectifs propres à chaque étape) qui lui permettent de progresser vers l'objectif de l'étape. Comme le déroulement de l'intervention est étroitement influencé par le contexte de l'entreprise, l'ordre ou le temps requis pour atteindre chacune des balises varie d'une intervention à l'autre. La figure 1 illustre les balises à chacune des étapes selon St-Vincent et al. (2011). Celles-ci seront décrites dans les prochains paragraphes. 
Figure 1 : Balises visées à chaque étape de la première phase de l'intervention (adaptation de StVincent et al., 2011).

Figure 1 : Sub-objectives aimed for each step of the ergonomic intervention first phase (adapted from StVincent et al., 2011)

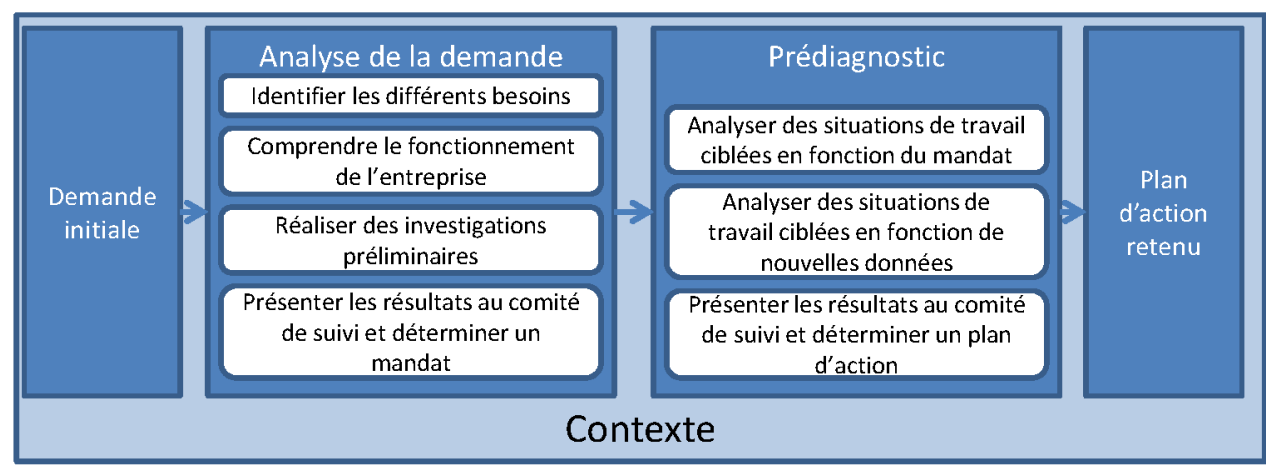

12 À la figure 1, on remarque qu'en plus de documenter les besoins et attentes entourant la demande initiale, l'intervenant peut chercher à recueillir les différents besoins exprimés par les acteurs de l'entreprise qui représentent des demandeurs ou des personnes concernées par la demande. Toujours à l'étape d'analyse de la demande, l'intervenant vise à comprendre le fonctionnement particulier de l'entreprise. Dans le cas spécifique des interventions étudiées, des informations sur différentes caractéristiques de l'entreprise sont colligées grâce à un outil nommé « Portrait de l'entreprise ». Ce document a été développé à partir d'études antérieures ayant documenté le contexte d'interventions dans les domaines de l'ergonomie (St-Vincent, Vézina, Laberge, Gonella, Lévesque, Petitjean-Roget, et al., 2010), de la santé au travail (Baril-Gingras, Bellemare, Poulin, \& Ross, 2010) et de la réadaptation (Durand, Corbiere, Coutu, Reinharz, \& Albert, 2014), ainsi que grâce à la consultation d'experts. Il permet de regrouper des informations touchant aux neuf catégories suivantes:

1. Caractéristiques générales de l'entreprise (taille, âge, secteur d'activité, etc.);

2. Caractéristiques de la population de travailleurs;

3. Situation économique ;

4. Représentation syndicale et relations de travail ;

5. Mode de gestion et organisation du travail ;

6. Projets en cours ou à venir ;

7. Prévention et santé/sécurité au travail (SST);

8. Gestion des invalidités et retour au travail et

9. Registres et statistiques.

Cette collecte de données se réalise auprès d'acteurs occupant des postes variés à différents niveaux hiérarchiques, ce qui permet également de réaliser une analyse stratégique permettant d'identifier le rôle de chaque acteur dans l'entreprise.

Dans le modèle de St-Vincent et al. (2011), on indique que l'intervenant réalise également des investigations préliminaires sur différentes situations de travail pour se familiariser avec l'activité réelle de travail et amorcer la sélection des situations les plus pertinentes à approfondir, que nous avons incluse dans l'analyse de la demande puisque cette étape n'est pas toujours distincte. Il peut également y avoir création de nouvelles données (ex. enquête, analyse de registres d'incidents/ accidents) en vue de faire un choix de situations à analyser. Toujours selon le modèle de St-Vincent et al. (2011), l'étape 
d'analyse de la demande se termine par une présentation formelle à un comité de suivi paritaire, c'est-à-dire formé de représentants des travailleurs et de l'employeur, qui devra s'entendre sur le mandat de l'intervention (objectif de l'étape), c'est-à-dire les situations à analyser plus en profondeur à la prochaine étape. Quand les acteurs-clés du comité de suivi sont d'accord sur le mandat, on considère que l'étape d'analyse de la demande est complétée.

14 À la figure 1, on remarque que lors de l'étape Prédiagnostic, l'intervenant réalise des analyses approfondies de situations de travail ciblées. Les situations peuvent avoir été ciblées lors de l'étape précédente ou émerger en fonction de nouvelles données recueillies à cette étape. En analysant l'activité de plusieurs travailleurs, l'intervenant cherche à identifier quels sont les déterminants à l'origine des situations critiques vécues par ces personnes, qui pourraient être modifiés et inclus dans le plan d'action pour améliorer la santé des travailleurs et la production. En connaissance du contexte, des contraintes et des possibilités de l'entreprise, l'intervenant présentera au comité de suivi les résultats de ses analyses (son " prédiagnostic ») pour discussion et négociation en vue du choix éclairé des projets de transformation à inclure dans le plan d'action (objectif de l'étape). Le prédiagnostic, suivant son acceptation ou incluant les ajustements qui y sont apportés, s'il y a lieu, grâce aux échanges avec les acteurs-clés, peut alors être considéré comme un diagnostic (St-Vincent et al., 2011). Quand les acteurs-clés du comité de suivi s'entendent sur le plan d'action à mettre en œuvre dans le cadre de l'intervention ergonomique, on considère qu'un diagnostic a été posé et que l'étape prédiagnostic est complétée.

En raison de la singularité du contexte de chaque entreprise, on peut s'attendre à ce qu'une intervention ergonomique donnée devienne comme une forme de produit unique qui ne fait que s'inspirer du modèle théorique initial décrit ci-haut. On envisage ainsi la possibilité d'adaptations des balises prévues, ou l'apparition de nouvelles balises, par exemple la mise sur pied précoce d'un projet de transformation à la demande d'acteursclés avant d'avoir complété l'étape Prédiagnostic. Ces variations découleront d'indicateurs de contexte qui pourront être mis en lumière grâce à l'évaluation des processus qui sera réalisée dans le cadre de cette étude.

\section{Méthode}

Cette évaluation des processus adopte le devis d'une étude de cas multiples (Stake, 2006). Le cas $(n=4)$ est défini comme une intervention ergonomique se déroulant dans une entreprise, du premier jour d'intervention dans l'entreprise jusqu'au moment où le plan d'action est négocié avec les acteurs-clés. Les quatre interventions évaluées (cas) ont eu lieu dans des entreprises de différents secteurs d'activité : transformation de légumes frais (cas A), emballage de produits pharmaceutiques (cas B), fabrication de comptoirs et de portes d'armoires (cas $C$ ) et transport de vrac par bateau (cas D). Le tableau 1 présente quelques caractéristiques structurelles des entreprises (Baril, \& Berthelette, 2000), dont le secteur d'activité, la taille de l'entreprise et la santé financière. De l'information plus précise sur la formulation des demandes d'intervention provenant de chaque entreprise se retrouve à la figure 3 (Section 4 Résultats). 
Tableau 1 : Caractéristiques structurelles des entreprises. Table 1: Structural characteristics of organizations

\begin{tabular}{|c|c|c|c|c|c|c|}
\hline Cas & $\begin{array}{c}\text { Secteur } \\
\text { d'activité } \\
\text { économique* }\end{array}$ & $\begin{array}{c}\text { Taille de } \\
\text { l'entreprise }\end{array}$ & $\begin{array}{c}\text { Santé } \\
\text { financière }\end{array}$ & $\begin{array}{c}\text { Présence } \\
\text { d'un } \\
\text { syndicat }\end{array}$ & $\begin{array}{c}\text { Présence d'un } \\
\text { comité de santé et } \\
\text { sécurité (paritaire) }\end{array}$ & $\begin{array}{c}\text { Programme } \\
\text { de } \\
\text { prévention }\end{array}$ \\
\hline A & Fabrication & Moyenne & Récession & Oui & Oui (oui) & Oui \\
\hline B & $\begin{array}{c}\text { Services } \\
\text { administratifs } \\
\text { et de soutien }\end{array}$ & Grande & Expansion & Non & Oui (oui) & Oui \\
\hline C & Fabrication & Grande & Récession & Non & Oui (oui) & Oui \\
\hline D & $\begin{array}{c}\text { Transport et } \\
\text { entreposage }\end{array}$ & $\begin{array}{c}\text { Très } \\
\text { grande }\end{array}$ & Expansion & Oui & Oui (non) & Non \\
\hline
\end{tabular}

* Selon le Système de classification des industries de l'Amérique du Nord (SCIAN)

\subsection{Interventions réalisées par des ergonomes émergents}

17 L'un des aspects de la complexité des interventions ergonomiques du courant de l'ergonomie de l'activité est leur grande diversité, qui peut se manifester tant en ce qui concerne le modèle théorique initial de l'intervenant, la nature de la demande, le type de relation avec le demandeur, les livrables, la durée, etc. Nous avons donc fait le choix de nous intéresser à une forme particulière d'intervention, qui présente certaines caractéristiques similaires. Il s'agit d'interventions réalisées par des ergonomes émergents (nommés ci-après $\mathrm{EE}$ ), c'est-à-dire des ergonomes en formation réalisant leur stage final menant au grade de maîtrise professionnelle en ergonomie dans une université québécoise. Le modèle théorique enseigné dans cette université québécoise est celui de St-Vincent et al. (2011) décrit à la section 2.2. Il constitue donc le modèle théorique initial commun à tous les EE dont les interventions sont évaluées dans notre étude. Par ailleurs, chaque EE intervient seul dans l'entreprise, tout en étant étroitement encadré à distance par une équipe de professeurs ergonomes pour assurer la qualité de l'intervention. En effet, chaque EE bénéficie d'un encadrement hebdomadaire formel à l'université, individuel et collectif, complété au besoin par des contacts avec les professeurs entre les séances d'encadrement formel. Une autre similarité entre les interventions évaluées relève de la demande initiale de chaque intervention, où chacune concerne la prévention des TMS chez les travailleurs (voir Figure 3 pour de l'information plus spécifique à ce sujet). La structure prévue de l'intervention est également la même, c'est-à-dire deux jours par semaine de présence dans l'entreprise, échelonnée sur un an. Environ six mois (soit l'équivalent d'environ 54 jours d'intervention) sont consacrés à la phase de développement de l'intervention, ou autrement dit aux étapes de l'intervention qui précèdent l'implantation des changements. Chaque entreprise s'engage à implanter des transformations au cours de la période prévue de stage, même si ceux-ci ne seront pas documentés dans le cadre de l'évaluation. Chaque entreprise doit également autoriser l'EE à accéder à des documents (ex. organigramme, plan de l'établissement, données statistiques d'accidents ou de production, etc.) ou à des personnes pouvant lui fournir l'information pour compléter le portrait de l'entreprise (décrit à la section 2.2), puisque l'un des objectifs pédagogiques est de bien cerner le contexte de l'intervention. 


\subsection{Collecte de données de l'évaluation}

18 La figure 2 illustre les moments de collecte des données (temps T1 à T4 sur la Figure 2), réalisée auprès des deux groupes de participants à l'évaluation, soit les $\mathrm{EE}$ (T1, T2 et T4) et les acteurs-clés impliqués dans le choix du plan d'action (T3). Pour chaque cas, les moments de collecte des données ont eu lieu le plus rapidement possible suivant la fin de chaque étape de l'intervention. Les méthodes, les sources et les moments de collecte des données ont également été choisis de façon à ce que l'évaluation interfère le moins possible avec le déroulement naturel de l'intervention (Lamonde, 2000 ; Landry, 2008).

Figure 2 : Aperçu des participants, moments et méthodes de collecte des données. Figure 2: Overview of participants, moments and methods for data collection

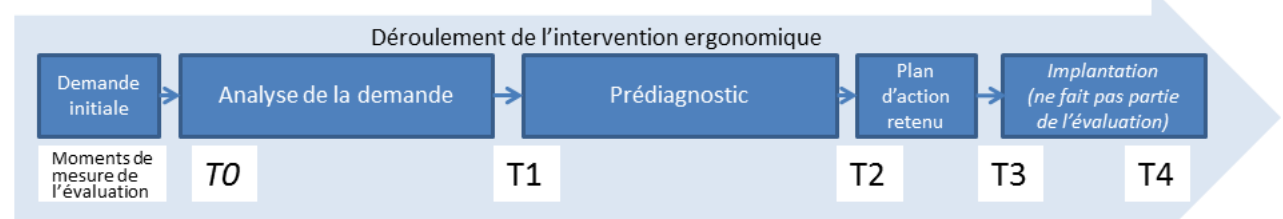

TO: Recrutement des EE et des entreprises

T1: Compilation du journal de bord, du portrait de l'entreprise et du rapport d'étape + Entretien semi-dirigé avec l'EE

T2: Compilation du journal de bord et du rapport d'étape + Entretien semi-dirigé avec l’EE

T3: Entretiens semi-dirigés ( $n=4-6$ par cas) avec les acteurs-clés impliqués dans le choix du plan d'action

T4: Entretien de validation avec l’EE (suivant l'analyse des données)

Conformément à des recommandations émises pour réaliser l'évaluation d'interventions ergonomiques (Landry, 2008), des documents produits dans le cadre de l'intervention ont été utilisés pour colliger les données liées aux contextes et aux processus. Le premier document retenu est un journal de bord complété de façon hebdomadaire par l'EE. Il s'agit d'une base de données informatisée qui contient, pour chaque action réalisée : la date, la durée, le type d'activité (ex. observation, entretien, recherche documentaire, etc.), l'acteur ou les acteurs rencontrés et un bilan de l'activité. Cet outil a été développé spécifiquement pour réaliser le suivi et l'évaluation d'interventions ergonomiques dans le cadre d'un projet de recherche antérieur (Vézina, Chatigny, Comtois, Durand, Fortin, Messing, et al., 2006) et a été prétesté dans le cadre d'une étudepilote pour s'assurer que son contenu convienne à une évaluation des processus (Albert, Vézina, Bilodeau, \& Coutarel, 2016). Les autres documents retenus sont le « Portrait de l'entreprise » complété par l'EE lors de l'analyse de la demande (décrit à la section 2) et des rapports d'étape, disponibles sous deux formes : la version papier de la présentation orale faite aux acteurs-clés du comité de suivi relatant les résultats de l'étape et un rapport écrit détaillé.

À la figure 2, on voit également que des entretiens semi-dirigés ont été réalisés par une même évaluatrice (VA) avec les EE et des acteurs-clés. Les entretiens semi-dirigés avec l'EE à T1 et T2 ont permis d'approfondir les données disponibles dans les documents concernant le déroulement de l'intervention et les indicateurs de contexte ayant influencé soit les stratégies d'intervention et les actions réalisées, soit le plan d'action. À $\mathrm{T} 3$, les entretiens avec les acteurs-clés ont notamment permis de trianguler les indicateurs de contexte ayant influencé les décisions entourant les projets retenus au plan d'action. Le tableau 2 présente les participants ( $\mathrm{H}$ : homme, $\mathrm{F}$ : femme) à l'évaluation qui ont été interrogés dans le cadre des entretiens semi-dirigés, pour un total de 
15 hommes et 7 femmes. Les acteurs-clés recrutés et interrogés ont tous été impliqués dans le choix du plan d'action. Ils ont été choisis en collaboration avec l'EE pour la complémentarité de leurs rôles ${ }^{2}$, offrant ainsi différentes perspectives par rapport à l'intervention. Soulignons par contre qu'il n'a pas été possible d'interroger un haut dirigeant pour le cas B ni un travailleur pour le cas D, en raison d'indicateurs de contexte propres à ces deux cas qui seront détaillés dans la section Résultats.

Tableau 2 : Participants interrogés pour chaque cas. Table 2 : Participants interviewed in each case

\begin{tabular}{|c|c|c|c|c|c|c|c|}
\hline \multirow[b]{2}{*}{ Cas } & \multirow[b]{2}{*}{$\mathrm{EE}$} & \multicolumn{5}{|c|}{ Catégorie d'acteurs-clés (AC) membres des comités de suivi } & \multirow{2}{*}{$\begin{array}{c}\text { Total } \\
\text { par } \\
\text { cas }\end{array}$} \\
\hline & & $\begin{array}{l}\text { Haute } \\
\text { direction }\end{array}$ & $\begin{array}{l}\text { Superviseur / } \\
\text { gestionnaire* }\end{array}$ & $\begin{array}{l}\text { Responsable } \\
\text { SST ou RH* }\end{array}$ & $\begin{array}{l}\text { Ingénieur/ } \\
\text { mécanicien* }\end{array}$ & $\begin{array}{l}\text { Travailleur / } \\
\text { représentant* }\end{array}$ & \\
\hline A & $\mathrm{H}$ & $2 \mathrm{H}$ & & $1 \mathrm{H}^{*}$ & $1 \mathrm{~F}^{*}$ & $1 \mathrm{H} / 1 \mathrm{H}^{*}$ & 7 \\
\hline $\mathrm{B}$ & $\mathrm{H}$ & & $1 \mathrm{H}$ & $1 \mathrm{H}$ & $1 \mathrm{H}$ & $1 \mathrm{~F}$ & 5 \\
\hline $\mathrm{C}$ & $\mathrm{F}$ & $1 \mathrm{~F}$ & $1 \mathrm{H}^{*}$ & $1 \mathrm{H}$ & & $1 \mathrm{~F}$ & 5 \\
\hline $\mathrm{D}$ & $\mathrm{F}$ & $1 \mathrm{~F} 1 \mathrm{H}$ & & & $2 \mathrm{H}$ & & 5 \\
\hline Total & 4 & 5 & 2 & 3 & 4 & 4 & 22 \\
\hline
\end{tabular}

21 De l'information additionnelle concernant le contenu du journal de bord et les guides d'entretiens sont disponibles dans une autre publication disponible en libre accès (Albert, Vézina, Bilodeau, \& Coutarel, 2017).

\subsection{Analyse des données}

L'analyse des données s'est réalisée en trois temps. Dans un premier temps, une analyse par cas a été réalisée suivant chaque étape de l'intervention. Une compilation quantitative du journal de bord (nombre de jours d'intervention, d'heures et d'actions réalisés, répartition des types d'actions réalisées et des catégories d'acteurs-clés rencontrés) a d'abord été produite pour obtenir un aperçu de la dynamique d'ensemble de chaque cas (Petit et al., 2007). Les résultats de cette analyse sont disponibles au tableau 3 (section 4. Résultats). Une compilation qualitative du journal de bord et du rapport d'étape a permis de préparer chaque entretien semi-structuré avec l'EE. Dans un deuxième temps, une analyse thématique des entretiens avec l'EE et les acteurs-clés a été réalisée selon une grille d'analyse composée de rubriques prédéfinies (contexte, stratégies d'intervention, actions déterminantes, acteurs-clés, processus de choix des projets, etc.), bonifiée de façon itérative des thèmes émergeant de l'analyse (Paillé, \& Mucchielli, 2003). Ces analyses ont été présentées à l'EE (T4, Figure 2) pour valider, modifier ou approfondir les résultats générés (Stake, 2006). Seules des modifications mineures ont été réalisées à la lumière de ces entretiens. Une triangulation des données obtenues grâce aux différentes sources a permis de produire un modèle logique (Brousselle et al., 2011) pour chaque cas. Les modèles logiques sont présentés à la figure 3 (section 4. Résultats). Dans un troisième temps, une analyse intercas a été réalisée grâce à la création d'une matrice (Stake, 2006) comparant les indicateurs du contexte initial ayant influencé chacun des cas. On considère qu'un indicateur de contexte a influencé l'intervention s'il est rapporté explicitement par au moins un participant (EE ou acteurclé). En effet, même si la présence de plus d'une source relatant l'influence d'un indicateur donné était systématiquement recherchée (Stake, 2006), on reconnaît à priori que les participants à l'évaluation peuvent avoir des perspectives différentes dues à leur rôle et leur niveau de connaissance d'enjeux particuliers dans l'entreprise. Dans notre 
approche, la triangulation est également utilisée pour révéler différentes façons de voir un même phénomène (Stake, 1994) et la diversité des acteurs interrogés dans le cadre de l'évaluation contribue à la richesse des perspectives disponibles. On retrouve les indicateurs de contexte à la figure 3 et au tableau 4 (section 4. Résultats).

\section{Résultats}

Les résultats de l'évaluation des processus détaillés dans les prochaines sections permettent de comparer les quatre interventions à l'étude, d'abord d'un point de vue global (dynamique d'ensemble des interventions). Puis, ils permettent plus spécifiquement de comparer les indicateurs de contexte initial et les différents types d'actions réellement engagées par les EE pour réagir aux indicateurs détectés ou pour changer le contexte d'intervention.

Tout d'abord, une comparaison de la dynamique d'ensemble de chaque intervention (cas) est disponible sous deux formes. Le tableau 3 présente des données quantitatives permettant de comparer les processus (actions réalisées par l'EE) pour chaque cas, soit le nombre de jours d'intervention précédant le plan d'action, la durée de chaque étape et la proportion totale d'interactions avec des travailleurs.

Tableau 3 : Comparaison des actions réalisées par l'EE pour chaque cas.

Table 3 : Comparison of emerging ergonomists' actions for each case

\begin{tabular}{|c|c|c|c|c|c|c|c|c|}
\hline \multirow[t]{2}{*}{ Cas } & \multicolumn{3}{|c|}{$\begin{array}{c}\text { Nombre de jours } \\
\text { d'intervention } \\
\text { précédant le plan d'action }\end{array}$} & \multicolumn{3}{|c|}{$\begin{array}{l}\text { Durée de l'intervention } \\
\text { précédant le plan d'action } \\
\text { (en heures) }\end{array}$} & \multicolumn{2}{|c|}{$\begin{array}{l}\text { Durée totale des } \\
\text { interactions avec } \\
\text { travailleurs }\end{array}$} \\
\hline & Total & $\mathrm{AD}(\%)$ & $\mathrm{P}(\%)$ & Total & $\mathrm{AD}(\%)$ & $\mathrm{P}(\%)$ & Heures & $\%$ total \\
\hline A $\Rightarrow$ & 42 & $13(31)$ & $29(69)$ & 93,0 & $24,3(26)$ & $68,6(74)$ & 59,8 & 64 \\
\hline $\mathrm{B}$ 을 & 32 & $21(66)$ & $11(34)$ & 91,7 & $54,9(60)$ & $36,8(40)$ & 63,9 & 70 \\
\hline $\mathrm{C}$ & 48 & $33(69)$ & $15(31)$ & 115,8 & $80,2(69)$ & $35,6(31)$ & 40,8 & 35 \\
\hline 悉 & 58 & $27(47)$ & $31(53)$ & 281,5 & $130,2(46)$ & $151,3(54)$ & 96,4 & 34 \\
\hline
\end{tabular}

AD : Analyse de la demande; P : Prédiagnostic

La figure 3 présente quant à elle les modèles logiques illustrant l'ordre de réalisation des balises (bulles blanches) de chaque étape. Les nouvelles balises sont identifiées par les bulles noires sur la figure 3. La longueur de chaque étape (représentées par les boîtes grises nommées Analyse de la demande et Prédiagnostic) est conforme à la proportion des durées retrouvées au tableau 3. On voit par exemple que l'analyse de la demande a été proportionnellement plus longue dans les cas $\mathrm{B}$ et $\mathrm{C}$, alors que le prédiagnostic a été plus long pour les cas $\mathrm{A}$ et $\mathrm{D}$, ce que l'on retrouve également au tableau 3. Sur la figure 3, les indicateurs de contexte initial ayant influencé au moins l'un des quatre cas sont représentés par des lettres correspondantes à la liste suivante :

a. Antécédent d'intervention ergonomique dans l'entreprise ;

b. Habitude de l'entreprise d'impliquer des travailleurs dans les projets ;

c. Attitude de certains acteurs de l'entreprise par rapport à l'intervention ;

d. Emplacement des travailleurs sur des sites en mouvement et éloignés du centre décisionnel ;

e. Présence d'intervenants externes lors des décisions concernant l'intervention ;

f. Mobilité des acteurs décisionnels à l'extérieur du site où a lieu l'intervention ; 
g. Variabilité de la production ;

h. Départs et arrivées d'acteurs décisionnels ;

i. Situation économique et disponibilité de ressources financières pour l'intervention ;

j. Disponibilité de ressources techniques pour les projets ;

k. Obligation légale de modifier un poste ou un équipement

1. Projets en cours ou déjà planifiés par l'entreprise. figure 3, afin d'expliquer l'influence positive (levier) ou négative (obstacle) des indicateurs de contexte sur les cas. Les indicateurs de contexte seront indiqués en caractères gras dans le texte pour faciliter leur repérage, puisqu'ils ne peuvent être traités de façon strictement séquentielle. En effet, il arrive qu'une combinaison d'indicateurs influence simultanément un cas. Des extraits verbatim permettront également d'illustrer l'impact de certains indicateurs sur un cas donné.

Figure 3 : Modèles logiques illustrant le déroulement de chaque cas. Figure 3: Logic models illustrating the sequence of events in each case

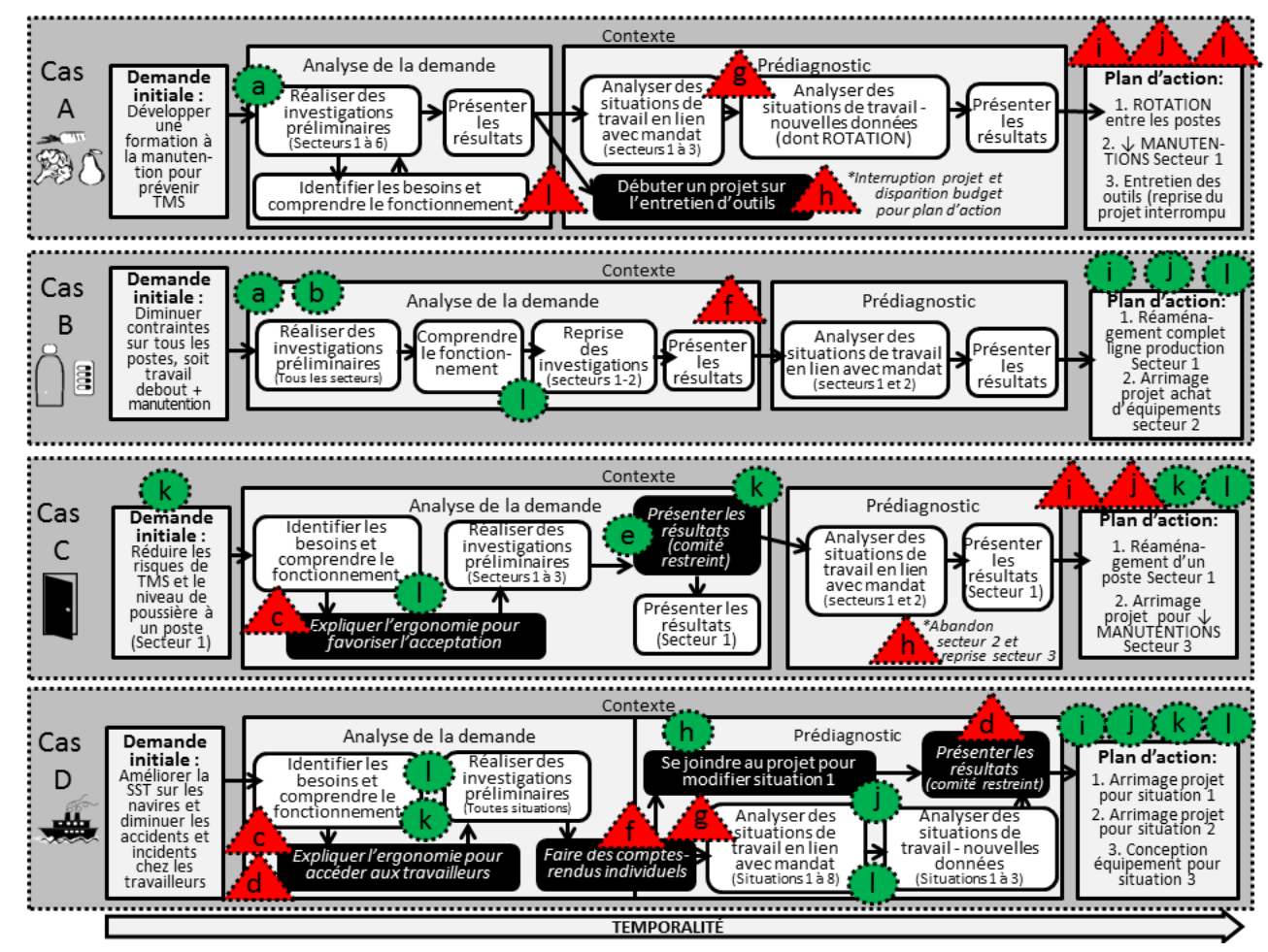

\subsection{Indicateurs de contexte lors de l'analyse de la demande}

L'antécédent d'intervention ergonomique dans l'entreprise (a; figure 3) est un premier indicateur de contexte ayant influencé les actions des EE au début de l'intervention. Les entreprises des cas $\mathrm{A}$ et $\mathrm{B}$ avaient déjà chacune bénéficié d'une intervention ergonomique de plus courte durée (a ; Figure 3), représentant un levier pour 
entrer en contact avec les travailleurs et réaliser des investigations préliminaires dès les premiers jours de l'intervention. De plus, spécifiquement pour le cas B, l'entreprise avait déjà l'habitude d'impliquer les travailleurs dans les projets de changement $(b$; Figure 3), représentant un levier additionnel pour réaliser les investigations préliminaires auprès des travailleurs. Cette combinaison d'indicateurs de contexte ( $a$ et $b$ ) peut d'ailleurs expliquer, du moins en partie, un résultat retrouvé au tableau 3, c'est-à-dire que le cas $B$ est celui où la plus grande proportion du temps d'intervention a été accordée aux interactions avec des travailleurs ( $70 \%$ du temps total d'intervention).

À l'opposé, les cas $\mathrm{C}$ et $\mathrm{D}$ n'avaient jamais bénéficié d'intervention ergonomique, ce qui pourrait expliquer l'attitude de certains acteurs envers l'intervention (c; Figure 3). Dans le cas $\mathrm{C}$, la réticence provenait des travailleurs, comme l'explique une travailleuse interrogée :

«Sur le coup, on disait: «Bon, une perdue.» On est tous une gang de vieux sceptiques sur mon bord (rire) [...] Elle m'a demandé si j'avais mal à des endroits, j'ai dit : « oui, oui, des fois. ». Mais t'sais j'ai été bien vague. Moi je suis une testeuse, je voulais juste tester ses connaissances. Parce que je me suis dit : «Avant de te faire confiance, je vais toujours bien savoir comment tu marches? » (C - Travailleuse)

Dans le cas $\mathrm{D}$, la réticence provenait plutôt d'acteurs décisionnels, comme l'explique un ingénieur interrogé :

«Pour être honnête au début, l'ergonomie, on ne connaît rien là-dedans. En la mettant sur le navire, on ne voulait pas qu'elle dérange les gens qui travaillent. Donc je pense qu'il y avait peut-être une petite... (silence) Une approche qu'on ne connaissait pas assez pour voir la contribution qu'elle aurait pu avoir. [...] On se méfiait un petit peu au début, je pense. » (D - Ingénieur)

31 Dans ces deux cas, l'attitude des acteurs envers l'intervention a représenté un obstacle à la réalisation d'investigations préliminaires auprès des travailleurs, qui représente une balise incontournable pour compléter l'analyse de la demande. Une stratégie d'intervention similaire a été adoptée par les deux EE faisant face à cet obstacle. En effet, de nombreuses actions additionnelles, comme des entretiens individuels auprès de gestionnaires et hauts dirigeants et la participation à des réunions de production ou SST, ont été réalisées par l'EE-C et l'EE-D pour construire un contexte favorable à la réalisation des investigations préliminaires. Ces actions sont regroupées à la figure 3 sous une nouvelle balise intitulée «Expliquer l'ergonomie ». L'EE-C explique la nécessité de cette nouvelle balise : «Je ne me sentais pas nécessairement à l'aise d'aller voir les travailleurs tant que je n'avais pas de lien avec les gestionnaires» (EE-C). Avant d'amorcer les investigations préliminaires avec les travailleurs, l'EE-C a donc adopté la stratégie d'interroger tous les gestionnaires de chaque secteur de l'entreprise pour identifier leurs besoins et obtenir de l'information sur des projets en cours prévus par l'entreprise ( 1 ; Figure 3) auxquels pourrait s'arrimer l'intervention.

Dans le cas $\mathrm{D}$, un indicateur de contexte additionnel a représenté un deuxième obstacle à la réalisation d'investigations préliminaires avec les travailleurs dans cette entreprise de transport, soit l'emplacement des travailleurs sur des sites en mouvement et éloignés du centre décisionnel ( $\mathrm{d}$; Figure 3). L'EE-D explique les difficultés rencontrées :

«C'était vraiment compliqué au début. Tout le monde se renvoyait la balle:

"Comment ça t'es pas allée sur un bateau? Tu aurais dû demander à telle personne." Ou : "Mais pourquoi tu n'es pas allée avec telle personne ?" » (EE-D)

En réaction à ces deux obstacles, la stratégie d'intervention mise en place par l'EE-D a été de progressivement identifier la chaîne d'acteurs décisionnels lui permettant de réaliser 
les investigations préliminaires et les analyses approfondies sur un navire non pas au port, comme l'entreprise avait l'habitude de procéder avec les contracteurs, mais bien sur un navire en déplacement, pour observer l'activité réelle des travailleurs. Il faut cependant souligner qu'au cours de ces nombreux entretiens, elle a été mise au courant d'un projet en cours ( 1 ; Figure 3 ) visant à modifier une situation de travail (situation 1 ) pour laquelle l'entreprise faisait également face à une obligation légale de modifier un équipement ( $\mathrm{k}$; Figure 3). L'EE-D a donc inclus cette situation de travail dans ses analyses, pour que celles-ci puissent contribuer à la modification de cet équipement, ce qui a représenté ultérieurement un levier pour l'inclure au plan d'action. Un arrimage précoce avec l'équipe d'ingénieurs responsables de ce projet a également eu lieu, soit avant que le plan d'action soit formellement établi, comme le montre la nouvelle balise lors du prédiagnostic (nommée « Se joindre au projet pour modifier situation 1 ») que l'on retrouve pour le cas D à la figure 3.

\subsection{Indicateurs de contexte lors des décisions prises concernant le mandat} l'intervention, comme au moment de la planification du mandat et du plan d'action, sont prises par un comité de suivi paritaire formé d'acteurs-clés (travailleurs et décideurs) internes à l'entreprise à la suite de la présentation des résultats de l'intervenant. Or, la figure 3 montre que des adaptations à cette procédure ont dû être faites dans les cas $\mathrm{C}$ et D.

Pour le cas $\mathrm{C}$, la présence d'intervenants externes lors de décisions à prendre concernant l'intervention (e ; Figure 3 ) a grandement influencé le choix du mandat lors de l'analyse de la demande, comme en témoigne la directrice $\mathrm{RH}$ :

"C'est [l'équipe d'intervenants externes de la santé publique] qui a certainement aidé à prioriser ce mandat-là [le secteur 1]. Et le faire accepter à l'ensemble des personnes décisionnelles dans l'entreprise. » (C - Directrice $\mathrm{RH})$

Il faut préciser que l'entreprise faisait face à une obligation légale de modifier un poste ( $\mathrm{k}$; Figure 3) du secteur 1 en raison d'un taux de poussières de bois hors norme identifié par ces mêmes intervenants de la santé publique et que cette obligation avait d'ailleurs fait émerger la demande initiale d'intervention ergonomique. Les intervenants externes avaient donc tenu à être impliqués lorsque les résultats de l'analyse de la demande seraient présentés aux dirigeants (nommé «comité restreint» sur la figure 3) pour s'assurer de réitérer l'importance d'analyser le secteur 1 et de l'inclure au plan d'action. L'EE-C explique d'ailleurs que les dirigeants ne considéraient pas le secteur 1 comme une priorité :

" Admettons, s'il n'y avait pas eu rien, pas de "hors-norme", pas rien. Pas sûre qu'ils auraient été d'accord [pour le secteur 1]. Parce qu'ils ont tellement d'autres priorités dans l'entreprise. Puis ils me l'ont fait sentir aussi que ce n'est pas làdessus qu'ils veulent que je travaille. » (EE-C)

En effet, deux autres secteurs (2 et 3) apparaissaient prioritaires pour les dirigeants en raison du nombre d'accidents et de la présence de projets déjà planifiés par l'entreprise auxquels l'intervention ergonomique pouvait s'arrimer (1; figure 3). En somme, pour le cas C, la collaboration entre l'EE-C et des intervenants externes a représenté une stratégie d'intervention intéressante pour appuyer la pertinence de 
modifier une situation de travail qui comportait des problèmes, mais qui n'étaient pas initialement considérés comme prioritaires par certains acteurs décisionnels de l'entreprise.

Pour le cas $\mathrm{D}$, des modifications à la structure participative prévue au modèle d'intervention de St-Vincent et al. (2011) ont eu lieu en raison de l'emplacement des travailleurs sur des sites en mouvement et éloignés du centre décisionnel $(d$; figure 3), mais également en raison de la mobilité des acteurs décisionnels à l'extérieur du site où a lieu l'intervention (f; Figure 3), due à leurs diverses obligations dans cette entreprise multinationale. D'une part, le fait que les travailleurs soient sur des navires, constamment éloignés du centre décisionnel et en mouvement a représenté un obstacle au respect de la structure participative prévue par le modèle d'intervention, en ce sens qu'il n'a pas permis de former un comité paritaire, c'est-à-dire formé de représentants des travailleurs et de l'employeur, pour assurer le suivi de l'intervention. Il n'a pas non plus été possible d'interroger des travailleurs pour l'évaluation dans le cas D, comme en témoigne le contenu du tableau 2 (section 3 Méthode). De plus, à l'étape d'analyse de la demande, certains membres de la haute direction qui devaient donner leur accord pour la progression de l'intervention étaient régulièrement à l'extérieur du site en raison d'obligations professionnelles. Cet indicateur a représenté un obstacle au déroulement de l'intervention. En effet, une présentation formelle des résultats de l'analyse de la demande en comité n'a pas pu être effectuée, en raison de l'impossibilité à rassembler tous les acteurs dans des délais raisonnables. Pour éviter de générer une période de latence dans l'intervention, l'EE-D a dû adopter la stratégie de réaliser plusieurs comptes rendus individuels aux membres de la haute direction devant donner leur accord à la poursuite des analyses approfondies de l'étape du prédiagnostic. La transition entre les deux étapes s'est donc faite de façon graduelle, comme en témoigne le chevauchement de ces étapes pour le cas D à la figure 3.

La mobilité des acteurs décisionnels à l'extérieur du site où a lieu l'intervention (f ; Figure 3) est un indicateur de contexte ayant représenté un obstacle pour le cas B également. La directrice de production était responsable de tous les sites de l'entreprise, donc très peu disponible ou présente sur le site où se déroulait l'intervention. Par conséquent, l'EE ne l'a rencontrée qu'une seule fois et très brièvement à l'extérieur des deux présentations formelles de résultats, de sorte qu'il a été difficile pour l'EE-B de cerner ses attentes et son rôle par rapport à l'intervention. Il n'a pas non plus été possible d'interroger cette directrice dans le cadre de l'évaluation, comme on peut le constater au tableau 2 (section 3 Méthode).

\subsection{Indicateurs de contexte lors du prédiagnostic}

Lors de l'étape du prédiagnostic, la variabilité de la production d'une journée à l'autre (g; Figure 3) a représenté un obstacle pour les cas A et D, en entraînant des difficultés à planifier les analyses approfondies des situations de travail. Cet indicateur de contexte peut expliquer la durée plus longue de cette étape pour les cas A et D, tel qu'on le retrouve au tableau 3 et à la figure 3 , par les boîtes plus longues pour l'étape du prédiagnostic. Pour le cas A, les entretiens avec les travailleurs étaient particulièrement difficiles à réaliser, puisque la planification était impossible, comme l'explique l'EE-A :

«Il y a tellement de la variabilité dans chaque journée. Il y a des journées qu'on me

disait: "Oui, je peux te rencontrer", mais "Finalement, non, peux-tu dans une demi- 
heure ?" [ou] "Non, non, je ne peux pas, parce qu'on est en manque de personnel." [...] Il n'y a pas d'heure fixe, je ne peux pas les rencontrer à telle heure, ou c'est assez rare qu'ils peuvent se libérer [...] C'est vraiment au jour le jour. » (EE-A) D étaient présents $24 \mathrm{~h} / 24$ sur le navire. Par contre, il était fréquent que la planification des tâches à réaliser sur le navire soit modifiée au cours d'une même journée en fonction des conditions climatiques, du déplacement du navire et des conventions collectives à respecter pour les heures de repos des travailleurs. L'EE-D explique : «Ce qui arrive, c'est que parfois, LÀ, il faut que ça soit fait. [...] J'essayais de planifier, mais un moment donné, au début je voulais, mais après ça [j'ai laissé tomber].» Pour surmonter cet obstacle et arriver à analyser toutes les situations de travail requises pour poser son prédiagnostic, l'EE-D a adopté comme stratégie de remettre régulièrement au capitaine une liste des situations à analyser et ce dernier ou l'un de ses officiers contactait l'EE-D pour l'aviser du début d'une tâche ou d'une modification à l'horaire prévu. La durée d'activités de l'EE-D lors des présences sur le navire pouvait d'ailleurs dépasser les 12 heures par jour, ce qui explique que le nombre d'heures totales pour cette intervention soit beaucoup plus élevé que les autres cas, comme en témoignent les données disponibles au tableau 3.

Un autre indicateur de contexte important concerne les départs et arrivées d'acteurs décisionnels (h ; Figure 3), qui sont survenus dans tous les cas évalués. Bien que l'impact ait été peu significatif pour le cas $\mathrm{B}$, cet indicateur a représenté un obstacle au plan d'action pour les cas A et C, mais un levier pour le cas D. Pour le cas C, le départ d'un haut dirigeant tout juste avant la sélection du plan d'action a entrainé l'abandon d'un projet planifié par l'entreprise au secteur 2 et la modification du plan d'action pour l'intervention ergonomique, comme le coordonnateur SST l'explique :

«Il y aurait eu un autre projet [au secteur 2]. [L'EE-C] avait même commencé à travailler dessus. Mais il y a eu des changements. La personne qui était responsable du dossier a quitté. Fait que là, on s'est rassis, on a rebrassé la soupe.» (C - Coordonnateur SST)

Les analyses réalisées par l'EE-C au secteur 2 n'ont donc pas pu être mises à profit pour améliorer les situations de travail dans ce secteur. Toutefois, le secteur 3, qui faisait partie des priorités de l'entreprise au moment de déterminer le mandat, mais non retenu à l'époque en raison de la présence et de l'influence d'intervenants externes de la santé publique, a finalement été réintégré au plan d'action en raison de l'arrimage possible de l'intervention ergonomique à un autre projet déjà planifié par l'entreprise (1; Figure 3) dans ce secteur.

Pour le cas A, le départ d'un haut dirigeant au cours de l'étape du prédiagnostic a entraîné l'annulation du budget mis de côté par ce dernier pour réaliser les transformations dans le cadre de l'intervention ergonomique et la suspension d'un projet de transformation mis sur pied de façon précoce dans l'intervention, tel que le montre la nouvelle balise pour le cas A à la figure 3 (« Débuter un projet sur l'entretien d'outils »). Ce départ a donc représenté un obstacle majeur au plan d'action du cas A.

À l'opposé, pour le cas $\mathrm{D}$, un changement de vice-présidence au tout début de l'étape de prédiagnostic a augmenté la priorité de la SST pour l'entreprise, représentant un levier pour le déroulement général de l'intervention à partir de ce moment. En effet, la nouvelle vice-présidente a offert son appui à la présence prolongée de l'EE-D sur le navire pour réaliser les analyses approfondies requises pour poser le prédiagnostic. Elle a également soutenu l'intégration de l'EE-D à des structures déjà existantes pour qu'un arrimage ait 
lieu entre l'intervention ergonomique et deux projets en cours menés par des équipes d'ingénieurs (voir section suivante pour plus d'information à cet effet).

\subsection{Indicateurs de contexte ayant influencé le plan d'action}

La situation économique de l'entreprise et la disponibilité de ressources financières pour l'intervention (i; Figure 3) ainsi que la disponibilité de ressources techniques pour réaliser les projets $(\mathrm{j}$; Figure 3 ) ont représenté un levier pour le plan d'action dans les cas B et D, mais un obstacle pour les cas A et C. En effet, les entreprises B et D étaient en pleine expansion au moment de l'intervention (voir Tableau 1). Pour ces deux cas, un budget était disponible pour réaliser des achats d'équipements et une équipe complète d'ingénieurs était sur place pour collaborer avec l'EE pour concevoir les nouvelles situations de travail. À l'opposé, les entreprises A et C étaient plutôt en situation de récession (Tableau 1), sans budget identifié pour l'intervention au moment d'établir le plan d'action. Ces deux entreprises ne disposaient pas non plus d'ingénieurs à l'interne et le secteur de la maintenance était surchargé d'autres projets, ne laissant que peu de temps pour l'intervention ergonomique, comme l'explique la mécanicienne du cas A: " On est tout le temps débordés. [...] C'est encore une histoire de budget, c'est tout le temps ça. C'est ça qui arrête [l'EE-A] dans ses projets. » (A - Mécanicienne). Ces deux obstacles identifiés pour les cas $\mathrm{A}$ et $\mathrm{C}$ ont fait en sorte que l'ampleur du plan d'action était plus limitée, faute de ressources temporelles et humaines allouables à l'intervention ergonomique.

À la figure 3, on constate également que les projets en cours ou déjà planifiés par l'entreprise ( 1 ; Figure 3$)$ ont représenté un obstacle additionnel à l'ampleur du plan d'action pour le cas A. En effet, de nombreuses modifications de machines avaient eu lieu au cours de l'année précédant l'intervention, suivant un accident grave, comme l'explique le directeur de la maintenance :

"Ça a été un gros plan d'action l'année passée, ça a duré quasiment toute l'année, beaucoup d'argent, beaucoup d'investissement de temps, de matériel, ça touchait chaque équipement, c'était du sur mesure partout. » (A - Directeur Maintenance)

Cette situation explique en partie la situation économique difficile (i; Figure 3 ) et la faible disponibilité de ressources humaines $(j$; Figure 3$)$ qui limitent les types de projets pouvant être inclus au plan d'action dans le cas A, comme l'explique le directeur Qualité :

« Des changements dans les équipements, ça aurait demandé du capital, mais ça n'aurait pas passé [au conseil d'administration], parce qu'on est en période de restrictions budgétaires. [...] Le critère principal pour la sélection de ces projets-là, c'est ceux où d'une part on avait le maximum de gains, avec le minimum d'implication du management. » (A - Directeur Qualité)

À l'opposé, les projets en cours ou déjà planifiés par l'entreprise (1; Figure 3$)$ ont représenté des leviers à l'ampleur du plan d'action pour les cas B, C et D. Des exemples pour les cas $\mathrm{C}$ et $\mathrm{D}$ ont déjà été fournis précédemment (sections 4.1 et 4.2). Pour le cas $\mathrm{B}$, des projets d'achats d'équipements étaient déjà planifiés, de sorte que les résultats présentés par l'EE-B concernant des besoins d'équipement sur certains postes ciblés au secteur 2 se sont concrétisés, améliorant ainsi plusieurs situations de travail. 


\subsection{Synthèse des indicateurs de contexte}

50 Le tableau 4 présente une synthèse des indicateurs de contexte initiaux ayant émergé de nos analyses, en précisant s'ils ont représenté un levier $(\bigcirc$; cercle vert) ou un obstacle $(\triangle$ ; triangle rouge) pour chaque cas. Dans cette section synthèse, nous ne reprendrons pas chacun des indicateurs identifiés, mais apporterons certaines précisions aux résultats présentés aux sections précédentes. Ces précisions visent notamment à éclairer la façon d'apprécier l'ampleur du plan d'action retenu ou encore de piloter l'intervention ergonomique.

Tableau 4 : Indicateurs de contexte initial pour les quatre cas évalués. Table 4: Initial context indicators for each case

\begin{tabular}{|c|c|c|c|c|}
\hline & $\begin{array}{c}\mathrm{A} \\
\text { 送 } \\
\end{array}$ & $\begin{array}{c}\mathrm{B} \\
\text { 角 } 1 \\
\end{array}$ & $\mathrm{C}$ & $\begin{array}{r}\mathrm{D} \\
\mathbf{3} \\
\end{array}$ \\
\hline a) Antécédent d'intervention ergonomique dans l'entreprise & 0 & P & & \\
\hline b) Habitude de l'entreprise d'impliquer des travailleurs dans les projets & & O & & \\
\hline c) Attitude de certains acteurs de l'entreprise par rapport à l'intervention & & & $\mathbf{A}$ & $\mathbf{A}$ \\
\hline $\begin{array}{l}\text { d) Emplacement des travailleurs sur des sites en mouvement et éloignés } \\
\text { du centre décisionnel }\end{array}$ & & & & $\Delta$ \\
\hline e) Présence d'intervenants externes lors des décisions à prendre & & & O & \\
\hline $\begin{array}{l}\text { f) Mobilité des acteurs décisionnels à l'extérieur du site où a lieu } \\
\text { l'intervention }\end{array}$ & & $\boldsymbol{\Delta}$ & & $\boldsymbol{\Delta}$ \\
\hline g) Variabilité de la production & $\Delta$ & & & $\Delta$ \\
\hline h) Départs et arrivées d'acteurs décisionnels & $\Delta$ & & $\Delta$ & 운 \\
\hline i) Situation économique et disponibilité de ressources financières & $\Delta$ & - & $\Delta$ & ? \\
\hline j) Disponibilité de ressources techniques pour réaliser les projets & $\Delta$ & O & $\Delta$ & - \\
\hline k) Obligation légale à modifier un poste ou un équipement & & & 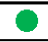 & ? \\
\hline 1) Projets en cours ou déjà planifiés par l'entreprise & $\Delta$ & 0 & 0 & 0 \\
\hline
\end{tabular}

Concernant le plan d'action, le tableau 4 permet de constater que les leviers $(\bigcirc$; cercle vert) sont particulièrement nombreux dans le cas $B$, alors que les obstacles $(\triangle$; triangle rouge) le sont davantage pour le cas A. Ceci peut contribuer à expliquer la variation dans la nature ou l'ampleur des plans d'action qui sont brièvement décrits à la figure 3. Par exemple, le projet de réaménagement complet d'une ligne de production dans le cas $\mathrm{B}$ n'aurait jamais été envisageable dans le cas A, compte tenu des obstacles identifiés, comme la faible disponibilité des ressources humaines et financières pour l'intervention. Nos résultats soulignent que dans le cadre d'une évaluation, on ne devrait pas juger de la valeur d'un plan d'action de prévention sans d'abord chercher à identifier les indicateurs de contexte initial auxquels l'intervenant a été confronté au cours de la phase de développement de l'intervention ergonomique. On ne peut pas non plus effectuer des comparaisons directes des plans d'action de différentes interventions en faisant abstraction des contextes. Ceci nous permet d'avancer l'idée que la valeur du plan d'action développé avec les acteurs-clés devrait nécessairement être appréciée en relation avec le contexte singulier de l'entreprise dans laquelle l'intervention a lieu.

L'influence des indicateurs de contexte initial identifiés dans chacun des cas s'est également manifestée sur le déroulement de l'intervention, notamment sur la capacité de l'EE à atteindre certaines balises requises pour compléter une étape ou encore sur les ressources temporelles (ou le nombre d'actions requises) pour compléter une étape. Par exemple, rappelons que l'attitude de certains acteurs envers l'intervention (cas $C$ et $D$ ) et l'emplacement des travailleurs sur des sites en mouvement et éloignés du centre 
décisionnel (cas $\mathrm{D}$ seulement) ont représenté des obstacles à la réalisation d'investigations préliminaires auprès de travailleurs qui sont requises pour compléter l'étape d'analyse de la demande. Ces indicateurs contribuent également à expliquer la proportion très faible d'interactions avec les travailleurs pour ces deux cas, comme on le retrouve au tableau 3. Si ces EE ont moins interagi avec les travailleurs, c'est parce qu'ils ont eu besoin de négocier davantage avec les autres acteurs (haute direction, gestionnaires, responsables SST ou RH par exemple), pour mettre en place des conditions favorables à la progression de l'intervention. En contrepartie, cette stratégie d'intervention impliquant de nombreux contacts avec les acteurs décisionnels en début d'intervention a permis aux EE C et D de prendre connaissance, de façon plus précoce que dans les deux autres cas, de projets planifiés par l'entreprise auxquels pourrait s'arrimer l'intervention, représentant ultérieurement un levier pour le plan d'action dans ces deux cas. Par conséquent, ces résultats issus des cas $C$ et $D$ suggèrent que de rechercher de façon précoce de l'information auprès de divers acteurs décisionnels sur les projets en cours ou déjà planifiés par l'entreprise serait une stratégie d'intervention à valoriser pour augmenter l'ampleur du plan d'action de prévention.

Concernant le pilotage de l'intervention, certains indicateurs de contexte initial identifiés dans cette étude pourraient être utiles à l'intervenant pour estimer la durée anticipée de l'intervention. En effet, des obstacles comme l'emplacement des travailleurs sur des sites en mouvement et éloignés du centre décisionnel (cas D), la mobilité des acteurs décisionnels à l'extérieur du site où a lieu l'intervention (cas D) et la variabilité de la production (cas A et D) ont augmenté les ressources temporelles requises pour compléter certaines balises de l'intervention, soit les investigations préliminaires et la détermination du mandat pour le cas $\mathrm{D}$, ainsi que les analyses approfondies pour les cas $\mathrm{A}$ et D. Par conséquent, l'identification précoce de tels indicateurs lors d'interventions futures pourrait permettre à l'ergonome de mieux anticiper les ressources temporelles requises pour compléter chaque étape de l'intervention, ou encore mieux justifier auprès des demandeurs la prévision du nombre d'heures d'intervention lors de l'entente de services.

\section{Discussion}

Cette étude présente les résultats d'une évaluation des processus d'interventions ergonomiques dont l'objectif était de dégager les indicateurs de contexte initial de l'entreprise qui ont influencé les actions de l'ergonome émergent ou le plan d'action négocié avec les acteurs-clés. Rappelons que nous qualifions le contexte d'« initial », car nos résultats montrent que certains indicateurs ont évolué au fil des interactions entre l'EE et les acteurs-clés, comme l'attitude de certains acteurs envers l'intervention.

À notre connaissance, il s'agit de la première évaluation des processus qui s'intéresse spécifiquement aux étapes qui précèdent l'implantation des changements dans le cadre d'interventions ergonomiques du courant de l'ergonomie de l'activité. Nos résultats montrent que ces étapes sont cruciales pour identifier des changements qui sont pertinents et surtout faisables pour une entreprise donnée. Dans les sections suivantes, nous discuterons d'abord des résultats, en rappelant certains indicateurs importants du contexte qui ont marqué le déroulement des interventions étudiées et les parallèles pouvant être faits entre nos résultats et d'autres études. Puis, nous discuterons des 
méthodes retenues ainsi que de l'apport de ce type de travaux au regard des modèles d'intervention qui ont cours dans la discipline.

\subsection{Discussion des résultats}

\subsubsection{Ressources financières}

Quelques évaluations des processus disponibles dans la littérature internationale ont identifié des obstacles à la phase d'implantation de changements dans le cadre d'une intervention ergonomique et l'obstacle le plus fréquemment rapporté est sans surprise le manque de ressources financières (Baumann et al., 2012 ; Pehkonen et al., 2009 ; van Eerd, Cole, Irvin, Mahood, Keown, Theberge, et al., 2010). Nos résultats montrent également que la disponibilité de ressources financières peut représenter un obstacle, mais le fait de détenir cette information avant de définir le plan d'action permet de choisir un plan d'action qui est cohérent avec les capacités et contraintes de l'entreprise, augmentant ainsi les probabilités que les modifications soient réellement implantées. Par exemple, dans le cas $\mathrm{A}$ où les restrictions financières étaient très sévères, le comité de suivi a opté pour un projet de rotation entre les postes (Plan d'action ; figure 3), qui ne représentait pas de coûts directs, mais qui pouvait tout de même améliorer les conditions de réalisation du travail. Par conséquent, la connaissance par l'intervenant et le comité de suivi des capacités et contraintes financières de l'entreprise avant de définir le plan d'action pourrait diminuer les difficultés d'implantation, en favorisant le choix de transformations mieux adaptées aux contraintes de l'entreprise, même si ces transformations ne sont pas optimales. En effet, limiter l'ambition de prévention peut représenter une stratégie d'intervention pour favoriser l'implantation de changements, puisque la proposition d'un plan d'action de prévention de grande envergure peut être rejetée par les acteurs-clés. En contrepartie, une trop grande censure peut faire en sorte que l'implantation de changements «minimaux» n'ait que peu d'impact sur la prévention des TMS. Le dosage du plan d'action apparaît donc comme un art, où l'intervenant devrait viser un maximum de prévention tout en demeurant dans le champ de l'acceptable pour l'entreprise. S'intéresser minutieusement au contexte de l'entreprise devient donc crucial pour atteindre cet objectif.

\subsubsection{Arrimage aux projets de changements planifiés par l'entreprise}

Deux études indiquaient que l'arrimage à des projets de rénovation, de construction, d'organisation de la production ou de changement technologique planifiés par l'entreprise peut représenter une occasion d'intégrer la prévention en santé et sécurité du travail (Robson, Amick, Moser, Pagell, Mansfield, Shannon, et al., 2016) et plus largement, la préoccupation pour l'activité réelle de travail (Baril-Gingras et al., 2010). Nos résultats abondent dans le même sens que ces études, où cet arrimage avec des projets déjà prévus par l'entreprise a représenté un levier dans trois cas sur quatre. Il semble donc s'agir d'une opportunité à exploiter pour augmenter la pertinence du plan d'action pour les acteurs, tout en requérant un minimum de ressources par rapport à ce qui avait déjà été prévu par l'entreprise. Nos résultats issus des cas $C$ et $D$ ajoutent également une notion de temporalité, où plus la connaissance des projets en cours ou à venir est acquise de façon précoce par l'ergonome, plus les investigations préliminaires et analyses approfondies peuvent s'orienter rapidement sur les situations de travail visées par les projets. Cette connaissance précoce permet à l'ergonome de mieux prioriser les 
situations de travail à analyser, ce qui peut contribuer à rendre l'intervention plus efficace et mieux ciblée sur les besoins et priorités de l'entreprise, tout en y intégrant les questions de prévention et la préoccupation pour l'activité réelle de travail.

\subsubsection{Instabilité des directions ou départs d'acteurs-clés}

L'instabilité des directions ou le départ d'acteurs-clés en cours d'intervention a été rapporté comme un obstacle à l'implantation de changements dans deux études (Caroly et al., 2008; van Eerd et al., 2010), la stabilité parmi les acteurs-clés étant habituellement recherchée pour faciliter l'implantation de changements d'envergure (Robson et al., 2016). Nos résultats abondent en ce sens pour les cas A et C. Toutefois, le départ et l'arrivée d'acteurs-clés se sont révélés un levier pour le déroulement de l'intervention et le plan d'action dans le cas $\mathrm{D}$, puisque l'acteur qui a quitté représentait un obstacle pour l'intervention. Nos résultats précisent donc que l'effet "personne» peut avoir son importance, puisque tout dépend de l'engagement envers l'intervention de l'acteur qui quitte l'entreprise ou y arrive. Il n'en demeure pas moins que l'intervenant a un rôle important à jouer lorsqu'un acteur en remplace un autre dans l'intervention. En effet, une étude mentionnait qu'il était fréquent d'assister à une faible transmission d'informations par rapport à l'intervention ergonomique entre le prédécesseur et le successeur (Caroly, Coutarel, Escriva, Roquelaure, Schweitzer, \& Daniellou, 2008). L'intervention ergonomique étant considérée comme une construction sociale collective (Daniellou, 2004), il semble que l'intervenant aurait intérêt à mettre à jour dès que possible le nouvel acteur (des étapes réalisées jusqu'à maintenant, des décisions prises, des contraintes dont on a tenu compte, etc.) pour diminuer les impacts potentiellement négatifs d'un tel changement.

\subsection{Discussion sur la méthodologie}

\subsubsection{Combinaison d'indicateurs}

59 Des liens émergent de nos résultats entre certains indicateurs de contexte, qui ont eu une influence synergique positive ou négative sur l'intervention. On peut souligner par exemple pour le cas D la combinaison d'obstacles concernant l'attitude initialement réticente d'acteurs décisionnels envers l'intervention et l'emplacement des travailleurs sur des sites en mouvement et éloignés du centre décisionnel qui ont fait en sorte qu'un très grand nombre d'heures d'intervention a été nécessaire pour arriver à réaliser les investigations préliminaires auprès de travailleurs lors de l'analyse de la demande. Un autre exemple, pour le cas $\mathrm{C}$, est la combinaison d'une obligation légale à modifier un poste et la présence d'intervenants externes qui ont représenté des leviers synergiques pour inclure une situation précise au plan d'action, laquelle ne faisait pas partie des priorités de l'entreprise. Cette émergence de configurations particulières d'indicateurs de contexte gagnerait d'ailleurs à être approfondie par des travaux futurs, car ces configurations représentent plus fidèlement la réalité et la complexité d'une entreprise qu'une série d'indicateurs isolés.

\subsubsection{Forces et limites}

60 Un certain nombre d'études en ergonomie ont mis en lumière l'influence d'indicateurs de contexte sur l'intervention, particulièrement sur la phase d'implantation des 
changements. Notre étude interdisciplinaire permet de bonifier cette compréhension en s'intéressant plus particulièrement à la phase de développement de l'intervention grâce aux modèles logiques qui donnent, d'une part, un aperçu du déroulement réel de chaque intervention, de l'ordre d'atteinte des balises (prévues et ajoutées) et qui illustrent, d'autre part, à quel moment dans l'intervention certains indicateurs de contexte ont eu une influence positive ou négative sur le déroulement de l'intervention ou le plan d'action. Cet apport au plan de la temporalité a été possible grâce à la collecte de données effectuée en temps réel par, entre autres, le journal de bord et sur une période prolongée, représentant une force de l'étude. Nos travaux ont également permis une description et une analyse en profondeur d'un petit nombre de cas, représentant une autre force de l'étude, en permettant de tirer de chacun des cas des propositions d'explications généralisables, qui fournissent des éclaircissements utiles pour des interventions futures (Shadish, Cook, \& Leviton, 1991). Les résultats présentés se concentrent principalement sur le contexte et certaines stratégies d'intervention réalisées par les EE en réponse aux indicateurs identifiés.

61 Concernant les actions, les résultats présentés demeurent tout de même un résumé qui ne rend pas compte de toute la complexité des actions ni des allers-retours qui ont lieu dans l'intervention ergonomique. Une limite de l'étude est d'ailleurs de ne pas avoir approfondi les multiples stratégies des ergonomes émergents. Selon le Centre National de Ressources Textuelles et Lexicales (CNRTL, 2012), une stratégie désigne un «ensemble d'actions coordonnées, d'opérations habiles, de manœuvres en vue d'atteindre un but précis ». Dans cette étude, nous nous sommes davantage concentrés sur la description des balises requises pour progresser à travers chacune des étapes de l'intervention, en offrant des exemples de stratégies réactives, adoptées par les EE pour surmonter les difficultés ou les obstacles. D'autres évaluations des processus selon le modèle proposé dans cette étude pourraient renseigner plus finement les stratégies d'intervention d'ergonomes chevronnés, puisque les outils de collecte des données utilisés dans cette étude le permettent. Ce faisant, on pourrait assister à l'émergence de stratégies non seulement réactives, mais également anticipatoires aux difficultés qui pourraient se manifester dans certains contextes, ce qui pourrait être d'une grande utilité pour bonifier les interventions futures.

62 Par ailleurs, il est possible que les indicateurs de contexte identifiés dans notre étude soient liés au modèle théorique initial des interventions ergonomiques retenues pour l'évaluation, à l'outil «Portrait de l'entreprise » utilisé ou au fait que les interventions soient réalisées par des ergonomes émergents. Ceux-ci pourraient ne pas avoir détecté la présence de certains indicateurs importants. Par contre, la triangulation des perspectives par l'inclusion d'acteurs-clés dans l'étude permet d'augmenter la robustesse des résultats présentés et la confiance dans le fait que les indicateurs importants pour les quatre cas à l'étude ont été identifiés. Il demeure tout de même essentiel de confronter les données obtenues dans le cadre de cette étude à des travaux futurs évaluant d'autres interventions ergonomiques du courant de l'ergonomie de l'activité pour élargir la portée de nos résultats. L'évaluation d'interventions ergonomiques reposant sur des modèles théoriques initiaux différents pourrait d'ailleurs mener à l'identification d'autres indicateurs de contexte, d'où la pertinence de reprendre ce type d'évaluation dans d'autres situations d'intervention. Pour ce faire, conformément aux directives produites par le Medical Research Council, il serait utile que les futures évaluations des processus 
d'interventions ergonomiques aient recours à des méthodes similaires à celles que nous avons développées pour favoriser les comparaisons entre les études (Moore et al., 2015).

\section{Conclusion}

Pour l'ergonome émergent, saisir les particularités du contexte d'une entreprise de façon à piloter l'intervention ergonomique et à négocier un plan d'action réaliste et faisable est une activité complexe qu'il devient utile de comprendre pour en tirer des leçons. Le modèle d'évaluation des processus présenté dans le cadre de cette étude a été développé sur des bases interdisciplinaires et sur mesure pour des interventions réalisées par des ergonomes émergents, mais pourrait être applicable à d'autres interventions ergonomiques complexes du courant de l'ergonomie de l'activité. Les résultats présentés fournissent des repères intéressants à la fois pour les ergonomes émergents et chevronnés, concernant les indicateurs de contexte initiaux à documenter pour mieux anticiper les difficultés ou l'allocation des ressources temporelles de l'intervention en fonction des indicateurs que l'on découvre dans une entreprise. Le modèle d'évaluation des processus décrit et mis en œuvre dans le cadre de cette étude interdisciplinaire pourrait également être utile à des chercheurs qui s'intéressent à comprendre les mécanismes d'actions d'interventions ergonomiques complexes. D'autres évaluations des processus selon un modèle similaire permettraient de bonifier la connaissance des indicateurs de contexte influençant l'intervention ergonomique dans différentes situations d'intervention.

\section{BIBLIOGRAPHIE}

Albert, V. (2018). Développement d'un modèle d'évaluation des processus d'interventions ergonomiques visant à prévenir les troubles musculosquelettiques et améliorer les situations de travail. Thèse de doctorat, Montréal, Université du Québec à Montréal.

Albert, V., Vézina, N., Bilodeau, H., \& Coutarel, F. (2016). Analyse des processus menant à des changements dans une entreprise du secteur aéronautique : vers un modèle d'évaluation des interventions ergonomiques. Relations industrielles / Industrial Relations, 71(4), 713-740.

Albert, V., Vézina, N., Bilodeau, H., \& Coutarel, F. (2017). Comment et pourquoi : proposition d'un modèle d'évaluation des processus de la phase de développement d'interventions ergonomiques. Cadre méthodologique d'une évaluation de la phase de développement d'interventions ergonomiques. PISTES, 19(3).

Baril-Gingras, G., Bellemare, M., Poulin, P., \& Ross, J. (2010). Conditions et processus de changement lors d'interventions externes en SST : Élaboration d'outils pour les praticiens. R-647, Montréal : IRSST.

Baril, R., \& Berthelette, D. (2000). Les composantes et les déterminants organisationnels des interventions de maintien du lien d'emploi en entreprises. RR-238, Montréal : Institut de recherche Robert-Sauvé en santé et sécurité du travail. 
Baumann, A., Holness, D. L., Norman, P., Idriss-Wheeler, D., \& Boucher, P. (2012). The Ergonomic Program Implementation Continuum (EPIC): integration of health and safety-a process evaluation in the healthcare sector. J Safety Res, 43(3), 205-213.

Berthelette, D., Bilodeau, H., \& Leduc, N. (2008). Pour améliorer la recherche évaluative en santé au travail. Santé Publique, 20 Suppl 3, S171-179.

Brousselle, A., Champagne, F., Contandriopoulos, A. P., \& Hartz, Z. (Eds.). (2011). L'évaluation: concepts et méthodes (2e éd.). Montréal, Canada : Presses de l'Université de Montréal.

Caroly, S., Coutarel, F., Escriva, E., Roquelaure, Y., Schweitzer, J.-M., \& Daniellou, F. (2008). La prévention durable des TMS, Quels freins? Quels leviers d'action? Recherche-action 2004-2007, France : Direction Générale du Travail

Chen, H. T. (2005). Practical Program Evaluation: Assessing and Improving Planning, Implementation and Effectiveness. Thousand Oaks, USA : Sage Publications.

CNRTL. (Ed.) (2012) Centre National de Ressources Textuelles et Lexicales. Nancy, France.

Cole, D., Wells, R. P., \& “the worksite upper extremity research group". (2002). Interventions for musculoskeletal disorders in computer-intense office work: a framework for evaluation. Work \& Stress, 16(2), 95-106.

Coutarel, F., Caroly, S., Vézina, N., \& Daniellou, F. (2015). Marge de manoeuvre situationnelle et pouvoir d'agir : des concepts à l'intervention ergonomique. Le travail humain, 78(1), 9-29.

Coutarel, F., \& Petit, J. (2015). Prevention of MSDs and the development of empowerment. In P. Falzon (Ed.), Constructive Ergonomics (pp. 189-204). Boca Raton, FL: CRC Press.

Coutarel, F., Vézina, N., Berthelette, D., Aublet-Cuvelier, A., Descatha, A., Chaissaing, K., et al. (2009). Orientations pour l'évaluation systématique des interventions visant la prévention des troubles musculo-squelettiques au travail. PISTES, 11(2), https://journals.openedition.org/ pistes/2349.

Craig, P., Dieppe, P., Macintyre, S., Michie, S., Nazareth, I., \& Petticrew, M. (2013). Developing and evaluating complex interventions: the new Medical Research Council guidance. Int J Nurs Stud, 50 (5), 587-592.

Dale, A. M., Jaegers, L., Welch, L., Gardner, B. T., Buchholz, B., Weaver, N., et al. (2016). Evaluation of a participatory ergonomics intervention in small commercial construction firms. Am J Ind Med, $59(6), 465-475$.

Daniellou, F. (2004). L'ergonomie dans la conduite de projets de conception des systèmes de travail. In P. Falzon (Éd.), Ergonomie (pp. 359-373). Paris : Presses Universitaires de France.

Daniellou, F., \& Rabardel, P. (2005). Activity-oriented approaches to ergonomics: some traditions and communities. Theor Issues in Ergon Sci, 6(5), 353-357.

Denis, D., St-Vincent, M., Imbeau, D., Jette, C., \& Nastasia, I. (2008). Intervention practices in musculoskeletal disorder prevention: a critical literature review. Appl Ergon, 39(1), 1-14.

Diabaté, M., Stock, S., Vézina, N., Laberge, M., Albert, V., Sinden, K., et al. (2016). A critical review of the literature on the effectiveness of ergonomic interventions in preventing work-related musculoskeletal disorders. Conféernce présentée à la 9th International Scientific Conference PREMUS, Toronto, Canada.

Driessen, M.T., Proper, K.I., Anema, J.R., Bongers, P.M., \& van der Beek, A.J. (2010). Process evaluation of a participatory ergonomics programme to prevent low back pain and neck pain among workers. Implement Sci, 5, 65. 
Durand, M. J., Corbiere, M., Coutu, M. F., Reinharz, D., \& Albert, V. (2014). A review of best workabsence management and return-to-work practices for workers with musculoskeletal or common mental disorders. Work, 48(4), 579-589.

Durlak, J. A., \& DuPre, E. P. (2008). Implementation matters: a review of research on the influence of implementation on program outcomes and the factors affecting implementation. Am J Community Psychol, 41(3-4), 327-350.

Falzon, P. (2013). Ergonomie constructive (1re éd.. éd.) : Paris : Presses Universitaire de France, [2013], ()2013.

Goldenhar, L. M., LaMontagne, A. D., Katz, T., Heaney, C., \& Landsbergis, P. (2001). The intervention research process in occupational safety and health: an overview from the National Occupational Research Agenda Intervention Effectiveness Research team. J Occup Environ Med, 43 (7), 616-622.

Guérin, F., Laville, A., Daniellou, F., Duraffourg, J., \& Kerguelen, A. (2007). Comprendre le travail pour le transformer. La pratique de l'ergonomie. France : ANACT.

Lamonde, F. (2000). L'intervention ergonomique un regard sur la pratique professionnelle. Toulouse : Octarès.

Landry, A. (2008). L'évaluation de l'intervention ergonomique : de la recherche évaluative à la proposition d'outils pour la pratique. Thèse de doctorat, Bordeaux, Université Victor Segalen Bordeaux 2.

Langley, A. (2009). Studying processes in and around organizations. In D. A. Buchanan \& A. Brynam (Eds.), The Sage Handbook of Organizational Reesearch Methods (pp. 409-429). Londres: Sage.

Linnan, L., \& Steckler, A. (2002). Process evaluation for Public Health Interventions and Research; an overview. In A. Streckler \& L. Linnan (Eds.), Process Evaluation for Public Health Interventions and Research (pp. 1-23). San Fransisco : Jossey-Bass.

Montmollin de, M. (1997). Vocabulaire de l'ergonomie (2e éd. rev. et augm.. éd.). Toulouse: Octarès. Moore, G. F., Audrey, S., Barker, M., Bond, L., Bonell, C., Hardeman, W., et al. (2015). Process evaluation of complex interventions: Medical Research Council guidance. BMJ, 350, h1258.

Nielsen, K., \& Randall, R. (2013). Opening the black box: Presenting a model for evaluating organizational-level interventions. European Journal of Work and Organizational Psychology, 22(5), 601-617.

Paillé, P., \& Mucchielli, A. (2003). L'analyse qualitative en sciences humaines et sociales. Paris : Arman Colin.

Pehkonen, I., Takala, E. P., Ketola, R., Viikari-Juntura, E., Leino-Arjas, P., Hopsu, L., et al. (2009). Evaluation of a participatory ergonomic intervention process in kitchen work. Appl Ergon, 40(1), 115-123.

Petit, J., Querelle, L., \& Daniellou, F. (2007). Quelles données pour la recherche sur la pratique de l'ergonome? Le travail humain, 70, 391-411.

Querelle, L., \& Thibault, J. F. (2007). La pratique de l'intervention d'ergonomes consultants : une approche réflexive orientée par les outils. Activités, 4(1), URL : https://journals.openedition.org/ activites/1458.

Robson, L. S., Amick, B., Moser, C., Pagell, M., Mansfield, E., Shannon, H. S., et al. (2016). Important factors in common among organizations making large improvement in OHS performance: Results of an exploratory multiple case study. Safety Science, 86, 211-227. 
Shadish, W. R., Cook, T. D., \& Leviton, L. C. (1991). Foundations of program evaluation theories of practice. Newbury Park, Calif: Newbury Park, Calif. Sage.

St-Vincent, M., Vézina, N., Bellemare, M., Denis, D., Ledoux, É., \& Imbeau, D. (Eds.). (2011). L'intervention en ergonomie. Québec : Éditions multimondes - IRSST.

St-Vincent, M., Vézina, N., Laberge, M., Gonella, M., Lévesque, J., Petitjean-Roget, T., et al. (2010). L'intervention ergonomique participative pour prévenir les TMS : ce qu'en dit la littérature francophone. R-667, Montéal: IRSST.

Stake, R. E. (1994). Case Studies. In N.K. Denzin \& Y.S. Lincoln (Eds.), Handbook of qualitative research (pp. 236-247). Thousand Oaks, CA: SAGE Publications.

Stake, R. E. (2006). Multiple case study analysis. New York: Guilford Press.

van Eerd, D., Cole, D., Irvin, E., Mahood, Q., Keown, K., Theberge, N., et al. (2010). Process and implementation of participatory ergonomic interventions: a systematic review. Ergonomics, 53 (10), 1153-1166.

Vézina, N., Chatigny, C., Comtois, A. S., Durand, M.-J., Fortin, S., Messing, K., et al. (2006). Développement d'un outil de suivi et d'évaluation de l'intervention ergonomique visant la prévention des troubles musculosquelettiques. Montréal : Instituts de Recherche en Santé du Canada.

Visser, S., van der Molen, H. F., Sluiter, J. K., \& Frings-Dresen, M. H. (2014). Guidance strategies for a participatory ergonomic intervention to increase the use of ergonomic measures of workers in construction companies: a study design of a randomised trial. BMC Musculoskelet Disord, 15, 132.

Vliet Vlieland, T. P. (2002). Managing Chronic Disease: Evidence-Based Medicine or Patient Centred Medicine? Health Care Analysis, 10(3), 289-298.

Worrall, J. (2007). Evidence in Medicine and Evidence-Based Medicine. Philosophical Compass, 2/6, 981-1022.

Yazdani, A., Neumann, W. P., Imbeau, D., Bigelow, P., Pagell, M., Theberge, N., et al. (2015). How compatible are participatory ergonomics programs with occupational health and safety management systems? Scand J Work Environ Health, 41(2), 111-123.

\section{NOTES}

1. Cette étape correspond au diagnostic de Guérin et al. (2007), c'est-à-dire que les analyses de l'intervenant sont finalisées et prêtes à être présentées aux acteurs-clés de l'entreprise de telle sorte qu'elles servent à l'établissement du plan d'action.

2. Les catégories d'acteurs-clés énoncées au tableau 2 correspondent aux profils suivants :

- Haute direction : personnel travaillant aux échelons supérieurs de la direction détenant un pouvoir pour allouer des ressources financières ou humaines requises pour la mise en œuvre du plan d'action ;

- Superviseur / gestionnaire : personnel responsable du poste de travail ou du secteur qui fera l'objet de modifications dans le cadre du plan d'action ;

- Responsable SST / RH: personnel habituellement chargé de la santé et sécurité dans l'entreprise ;

- Ingénieur / mécanicien : personnel habituellement chargé de réaliser les modifications du travail ;

- Travailleur / représentant : opérateur directement concerné par la situation de travail analysée ou représentant syndical détenant une connaissance du travail réel 


\section{RÉSUMÉS}

Bien qu'il soit généralement convenu que les ergonomes adaptent leur intervention au contexte de chaque entreprise, la façon précise dont ils y parviennent et sur quels critères cette adaptation se base sont peu renseignés au plan scientifique. Une évaluation des processus a été réalisée en adoptant un devis d'étude de cas multiple, en vue d'identifier les indicateurs de contexte ayant influencé quatre interventions du courant de l'ergonomie de l'activité, réalisées par des ergonomes en formation. L'évaluation a porté sur les étapes de l'intervention précédant l'implantation des changements, soit de l'analyse de la demande jusqu'au moment où le plan d'action est convenu avec les acteurs-clés. La collecte des données a été effectuée grâce à un journal de bord, des documents produits dans le cadre de l'intervention, ainsi que des entretiens avec les intervenants et les acteurs-clés impliqués dans l'intervention. Nos résultats présentés sous forme de modèles logiques font ressortir 12 indicateurs de contexte ayant représenté des leviers ou des obstacles au déroulement de l'intervention ou au plan d'action. Des combinaisons d'indicateurs ont eu un effet synergique positif ou négatif sur les cas. Le modèle d'évaluation proposé dans cette étude interdisciplinaire pourrait être repris lors d'études subséquentes pour bonifier la compréhension de l'influence du contexte dans d'autres situations d'interventions ergonomiques.

Although a consensus exists on the fact that ergonomists adapt the intervention to each organization's context, the precise way in which this adaptation is performed and its criteria have been overlooked in the scientific literature. A process evaluation was performed using a multiple case study design, in order to identify context indicators influencing four interventions adopting an activity-oriented approach to ergonomics and performed by ergonomists in training. The evaluation focussed on the steps preceding the implementation of work modifications, namely from request analysis until an action plan was adopted by key stakeholders. Data collection methods included a logbook, progress reports and interviews with ergonomists in training and key stakeholders. Our results presented in logic models show that 12 context indicators were facilitators or obstacles to the progression of the intervention or the action plan. Some combinations of indicators had a positive or negative synergistic effect on the cases. The process evaluation model developed for this interdisciplinary study could be used in future studies to get a better understanding of how the organization's context influences other kind of ergonomic interventions.

\section{INDEX}

Keywords : evaluation research, complex intervention, ergonomics, musculoskeletal disorders

Mots-clés : recherche évaluative, intervention complexe, ergonomie, troubles musculosquelettiques 


\section{AUTEURS}

\section{VALÉRIE ALBERT}

Université du Québec à Montréal, Pavillon des sciences biologiques, 141 Président-Kennedy, Local SB-4294, Montréal QC CANADA, H2X 1Y4, albv1010@gmail.com

\section{NICOLE VÉZINA}

Université du Québec à Montréal, Pavillon des sciences biologiques, 141 Président-Kennedy, Local SB-4294, Montréal QC CANADA, H2X 1Y4, vezina.nicole@uqam.ca

\section{HENRIETTE BILODEAU}

Université du Québec à Montréal, École des sciences de la gestion, 315 Ste-Catherine Est, Montréal QC CANADA, H2X 3X2, bilodeau.henriette@uqam.ca

\section{FABIEN COUTAREL}

Université Blaise Pascal, Clermont-Ferrand 2, BP 104 - 63172 Aubière Cedex, FRANCE, Fabien.Coutarel@univ-bpclermont.fr 